\title{
Numerical Investigations on Several Stabilized Finite Element Methods for the Stokes Eigenvalue Problem
}

\author{
Pengzhan Huang, ${ }^{1}$ Yinnian He, ${ }^{1,2}$ and Xinlong Feng ${ }^{1}$ \\ ${ }^{1}$ College of Mathematics and System Sciences, Xinjiang University, Urumqi 830046, China \\ ${ }^{2}$ Faculty of Science, Xi'an Jiaotong University, Xi'an 710049, China
}

Correspondence should be addressed to Yinnian He, heyn@mail.xjtu.edu.cn

Received 27 May 2011; Accepted 3 July 2011

Academic Editor: Alexander P. Seyranian

Copyright (C) 2011 Pengzhan Huang et al. This is an open access article distributed under the Creative Commons Attribution License, which permits unrestricted use, distribution, and reproduction in any medium, provided the original work is properly cited.

Several stabilized finite element methods for the Stokes eigenvalue problem based on the lowest equal-order finite element pair are numerically investigated. They are penalty, regular, multiscale enrichment, and local Gauss integration method. Comparisons between them are carried out, which show that the local Gauss integration method has good stability, efficiency, and accuracy properties, and it is a favorite method among these methods for the Stokes eigenvalue problem.

\section{Introduction}

It is well known that numerical approximation of eigenvalue problems plays an important role in the analysis of the stability of nonlinear PDEs. Meanwhile, they are wildly used in many application areas: structural mechanics and fluid mechanics (see [1]). Thus, development of an efficient and effective computational method for investigating these problems has practical significance and has drawn the attention of many peoples. At the time of writing, numerous works are devoted to these problems (see [2-9], and the references cited therein). Yin et al. [10] derived a general procedure to produce an asymptotic expansion for eigenvalues of the Stokes problem on rectangular mesh. Chen and Lin [11] proposed the stream function-vorticity-pressure method for the eigenvalue problem. Rate of convergence estimates were derived for the approximation of eigenvalues and eigenvectors by Mercier et al. [12]. $\mathrm{Xu}$ and Zhou [13] proposed a two-grid discretization scheme for solving eigenvalue problems. 
Mixed finite element methods are a natural choice for solving fluid mechanics equations because these equations naturally appear in mixed form in terms of velocity and pressure $[14,15]$. In the analysis and practice of employing mixed finite element methods in solving the Stokes equations, the inf-sup condition has played an important role because it ensures a stability and accuracy of the underlying numerical schemes. A pair of finite element spaces that are used to approximate the velocity and the pressure unknowns are said to be stable if they satisfy the inf-sup condition. Intuitively speaking, the inf-sup condition is something that enforces a certain correlation between two finite element spaces so that they both have the required properties when employed for the Stokes equations. However, due to computational convenience and efficiency in practice, some mixed finite element pairs which do not satisfy the inf-sup condition are also popular. Thus, much attention has been paid to the study of the stabilized method for the Stokes problem.

Recent studies have focused on stabilization techniques, which include penalty [1618], regular [19], multiscale enrichment [20], and local Gauss integration method [21, 22]. There exist a lot of theoretical results for the stabilized mixed finite element methods for the Stokes equations, and the comparisons between them are also given (see [17, 19, 20, 22-24], and the references cited therein). In this paper, we mainly focus on the Stokes eigenvalue problem solved by these stabilized finite element methods based on the lowest equal-order finite element space pair. Moreover, we present the comparisons between these methods for the considered problem.

The remainder of this paper is organized as follows. In the Section 2, we introduce the studied Stokes eigenvalue problem, the notations, and some well-known results used throughout this paper. Then several stabilized mixed finite element methods are reviewed, and their key stabilization techniques are recalled in Section 3. Comparisons between these stabilized methods are performed numerically in Section 4. Finally, we end with some short conclusions in Section 5.

\section{Preliminaries}

Let $\Omega$ be a bounded, convex, and open subset of $\mathbb{R}^{2}$ with a Lipschitz continuous boundary $\partial \Omega$. We consider the Stokes eigenvalue problem as follows. Find $(u, p ; \lambda)$, such that

$$
\begin{gathered}
-v \Delta u+\nabla p=\lambda u \quad \text { in } \Omega, \\
\operatorname{div} u=0 \quad \text { in } \Omega, \\
u=0 \quad \text { on } \partial \Omega,
\end{gathered}
$$

where $u=\left(u_{1}(x), u_{2}(x)\right)$ represents the velocity vector, $p=p(x)$ the pressure, $v>0$ the viscosity, and $\lambda \in \mathbb{R}$ the eigenvalue.

We will introduce the following Hilbert spaces:

$$
X=H_{0}^{1}(\Omega)^{2}, \quad Y=L^{2}(\Omega)^{2}, \quad M=L_{0}^{2}(\Omega)=\left\{q \in L^{2}(\Omega): \int_{\Omega} q \mathrm{~d} x=0\right\} .
$$

The spaces $L^{2}(\Omega)^{m}, m=1,2$, are equipped with the $L^{2}$-scalar product $(\cdot, \cdot)$ and $L^{2}$-norm $\|\cdot\|_{L^{2}}$ or $\|\cdot\|_{0}$. The space $X$ is endowed with the usual scalar product $(\nabla u, \nabla v)$ and the norm $\|\nabla u\|_{0}$. 
Standard definitions are used for the Sobolev spaces $W^{m, p}(\Omega)$, with the norm $\|\cdot\|_{m, p}, m, p \geq 0$. We will write $H^{m}(\Omega)$ for $W^{m, 2}(\Omega)$ and $\|\cdot\|_{m}$ for $\|\cdot\|_{m, 2}$.

We define the continuous bilinear forms $a(\cdot, \cdot)$ and $d(\cdot, \cdot)$ on $X \times X$ and $X \times M$, respectively, by

$$
\begin{gathered}
a(u, v)=v(\nabla u, \nabla v), \quad \forall u, v \in X, \\
d(v, q)=(q, \operatorname{div} v), \quad \forall v \in X, \quad \forall q \in M,
\end{gathered}
$$

and a generalized bilinear form $B((\cdot, \cdot) ;(\cdot, \cdot))$ on $(X \times M) \times(X \times M)$ by

$$
B((u, p) ;(v, q))=a(u, v)-d(v, p)-d(u, q), \quad \forall(u, p),(v, q) \in X \times M .
$$

With the above notations, the variational formulation of problem (2.1) reads as follows. Find $(u, p ; \lambda) \in(X \times M) \times \mathbb{R}$ with $\|u\|_{0}=1$, such that for all $(v, q) \in X \times M$,

$$
B((u, p) ;(v, q))=\lambda(u, v) .
$$

Moreover, the bilinear form $d(\cdot, \cdot)$ satisfies the inf-sup condition [25] for all $q \in M$ :

$$
\sup _{v \in X} \frac{|d(v, q)|}{\|\nabla v\|_{0}} \geq \beta_{1}\|q\|_{0}
$$

where $\beta_{1}$ is a positive constant depending only on $\Omega$. Therefore, the generalized bilinear form $B((\cdot, \cdot) ;(\cdot, \cdot))$ satisfies the continuity property and inf-sup condition:

$$
\begin{gathered}
|B((u, p) ;(v, q))| \leq c\left(v\|\nabla u\|_{0}+\|p\|_{0}\right)\left(\|\nabla v\|_{0}+\|q\|_{0}\right), \quad \forall(u, p),(v, q) \in X \times M, \\
\sup _{(v, q) \in X \times M} \frac{|B((u, p) ;(v, q))|}{\|\nabla v\|_{0}+\|q\|_{0}} \geq \beta_{2}\left(v\|\nabla u\|_{0}+\|p\|_{0}\right), \quad \forall(u, p) \in X \times M,
\end{gathered}
$$

where $c$ and $\beta_{2}$ are the positive constants depending only on $\Omega$.

\section{Stabilized Mixed Finite Element Methods}

For $h>0$, we introduce the finite-dimensional subspaces $X_{h} \times M_{h} \subset X \times M$, which are characterized by $K_{h}$, a partitioning of $\Omega$ into triangles $K$ with the mesh size $h$, assumed to be uniformly regular in the usual sense.

Then we define

$$
\begin{aligned}
& X_{h}=\left\{u \in C^{0}(\bar{\Omega})^{2} \cap X:\left.u\right|_{K} \in P_{1}(K)^{2}, \forall K \in K_{h}\right\}, \\
& M_{h}=\left\{q \in C^{0}(\bar{\Omega}) \cap M:\left.q\right|_{K} \in P_{1}(K), \forall K \in K_{h}\right\},
\end{aligned}
$$

where $P_{1}(K)$ represents the space of linear functions on $K$. 
Remark 3.1 (Nonconforming finite element space). Denote the boundary edge by $\Gamma_{j}=\partial \Omega \cap$ $\partial K_{j}$ and the interior boundary by $\Gamma_{j k}=\Gamma_{k j}=\partial K_{j} \cap \partial K_{k}$. Set the centers of $\Gamma_{j}$ and $\Gamma_{j k}$ by $\zeta_{j}$ and $\zeta_{j k}$, respectively. The nonconforming finite element space $\mathrm{NC}_{h}$ for the velocity will be taken to be

$$
\mathrm{NC}_{h}=\left\{v: v_{j}=\left.v\right|_{K_{j}} \in P_{1}\left(K_{j}\right)^{2}, v_{j}\left(\zeta_{j k}\right)=v_{k}\left(\zeta_{k j}\right), v\left(\zeta_{j}\right)=0, \quad K_{j} \in K_{h}, \forall j, k\right\},
$$

where $P_{1}\left(K_{j}\right)$ is the set of all polynomials on $K_{j}$ of degree less than 1 . Note that the nonconforming finite element space $\mathrm{NC}_{h}$ is not a subspace of $X$. In this nonconforming case, the pair of finite element spaces is $\mathrm{NC}_{h} \times M_{h}$ [26]; that is, the conforming space is still used for pressure.

Moreover, the discrete mixed finite element formulation for the Stokes eigenvalue problem reads: find $\left(u_{h}, p_{h} ; \lambda_{h}\right) \in\left(X_{h} \times M_{h}\right) \times \mathbb{R}$ with $\left\|u_{h}\right\|_{0}=1$, such that for all $(v, q) \in$ $X_{h} \times M_{h}$

$$
B\left(\left(u_{h}, p_{h}\right) ;(v, q)\right)=\lambda_{h}\left(u_{h}, v\right) .
$$

Next, we denote by $U$ the array of the velocity and by $P$ the array of the pressure. It is easy to see that (3.3) can be written in matrix form:

$$
\left[\begin{array}{ll}
A & B \\
B^{T} & 0
\end{array}\right]\left[\begin{array}{l}
U \\
P
\end{array}\right]=\lambda_{h}\left[\begin{array}{ll}
E & 0 \\
0 & 0
\end{array}\right]\left[\begin{array}{l}
U \\
P
\end{array}\right],
$$

where the matrices $A, B$, and $E$ are deduced in the usual manner, using the bases for $X_{h}$ and $M_{h}$, from the bilinear forms $a(\cdot, \cdot), d(\cdot, \cdot)$, and $(\cdot, \cdot)$, respectively, and $B^{T}$ is the transpose of matrix $B$.

Remark 3.2. In the nonconforming case, we define the bilinear forms

$$
a^{\prime}(u, v)=v \sum_{K \in K_{h}}(\nabla u, \nabla v)_{K^{\prime}} \quad d^{\prime}(v, p)=\sum_{K \in K_{h}}(\operatorname{div} v, q)_{K^{\prime}}
$$

and a generalized bilinear form $B^{\prime}((\cdot, \cdot) ;(\cdot, \cdot))$ on $(X \times M) \times(X \times M)$ by

$$
B^{\prime}((u, p) ;(v, q))=a^{\prime}(u, v)-d^{\prime}(v, p)-d^{\prime}(u, q), \quad \forall(u, p),(v, q) \in X \times M
$$

Accordingly, the discrete nonconforming formulation for the Stokes eigenvalue problem is: find $\left(u_{h}, p_{h} ; \lambda_{h}\right) \in\left(\mathrm{NC}_{h} \times M_{h}\right) \times \mathbb{R}$ with $\left\|u_{h}\right\|_{0}=1$, such that for all $(v, q) \in \mathrm{NC}_{h} \times M_{h}$,

$$
B^{\prime}\left(\left(u_{h}, p_{h}\right) ;(v, q)\right)=\lambda_{h}\left(u_{h}, v\right) .
$$


Note that the lowest equal-order pair does not satisfy the discrete inf-sup condition:

$$
\sup _{v_{h} \in X_{h}} \frac{d\left(v_{h}, q_{h}\right)}{\left\|\nabla v_{h}\right\|_{0}} \geq \beta_{3}\left\|q_{h}\right\|_{0^{\prime}} \quad \text { or } \sup _{v_{h} \in \mathrm{NC}_{h}} \frac{d^{\prime}\left(v_{h}, q_{h}\right)}{\left\|\nabla v_{h}\right\|_{0, h}} \geq \beta_{3}\left\|q_{h}\right\|_{0^{\prime}} \quad \forall q_{h} \in M_{h}
$$

where the constant $\beta_{3}>0$ is independent of $h$, and $\left\|\nabla v_{h}\right\|_{0, h}$ is the discrete energy seminorm in the nonconforming case. In order to fulfill this condition, several ways have been used to stabilize the lowest equal-order finite element space pair.

Method 1 (Penalty method). Find $\left(u_{h}, p_{h} ; \lambda_{h}\right) \in\left(X_{h} \times M_{h}\right) \times \mathbb{R}$ with $\left\|u_{h}\right\|_{0}=1$, such that for all $(v, q) \in X_{h} \times M_{h}$,

$$
B\left(\left(u_{h}, p_{h}\right) ;(v, q)\right)+\frac{\varepsilon}{v}\left(p_{h}, q\right)=\lambda_{h}\left(u_{h}, v\right),
$$

where $\varepsilon>0$ is a penalty parameter. The performance of this method obviously depends on the choice of the penalty parameter $\varepsilon$. Then the matrix form of (3.9) can be expressed as

$$
\left[\begin{array}{cc}
A & B \\
B^{T} & \frac{\varepsilon}{v} D
\end{array}\right]\left[\begin{array}{l}
U \\
P
\end{array}\right]=\lambda_{h}\left[\begin{array}{ll}
E & 0 \\
0 & 0
\end{array}\right]\left[\begin{array}{l}
U \\
P
\end{array}\right],
$$

where the matrix $D$ is deduced in the usual manner, using the base for $M_{h}$, from $\left(p_{h}, q\right)$. Because the coefficient matrix of (3.10) is usually large and sparse, it is not easy to compute the numerical solution using a direct method. In general, one can use the Uzawa algorithm. By some simple calculation, we get

$$
E_{i+1}^{P}=\left(I-\tau\left(B^{T}\left(A-\lambda_{h} E\right)^{-1} B-\frac{\varepsilon}{v} D\right)\right) E_{i}^{P},
$$

where $E_{i}^{P}=P-P_{i}$ and $\tau$ is a positive constant.

Method 2 (Regular method). Find $\left(u_{h}, p_{h} ; \lambda_{h}\right) \in\left(X_{h} \times M_{h}\right) \times \mathbb{R}$ with $\left\|u_{h}\right\|_{0}=1$, such that for all $(v, q) \in X_{h} \times M_{h}$,

$$
B\left(\left(u_{h}, p_{h}\right) ;(v, q)\right)-\delta \sum_{K \in K_{h}}\left(\nabla p_{h}-\lambda_{h} u_{h}, \nabla q\right)_{K}=\lambda_{h}\left(u_{h}, v\right),
$$

where $\delta=h^{2} /(\alpha v)$ is a stabilization parameter and $\alpha>0$. The regular method uses a simple way to stabilize the mixed finite element approximation without a loss of accuracy. In fact, it can be treated the regular method as a special Douglas-Wang's scheme [19]. Then the matrix form of this stabilized version can be expressed as

$$
\left[\begin{array}{cc}
A & B \\
B^{T} & -\delta D_{1}
\end{array}\right]\left[\begin{array}{l}
U \\
P
\end{array}\right]=\lambda_{h}\left[\begin{array}{ll}
E & 0 \\
F & 0
\end{array}\right]\left[\begin{array}{l}
U \\
P
\end{array}\right]
$$


where additional blocks $D_{1}$ and $F$ correspond to the following respective terms:

$$
\sum_{K \in K_{h}}\left(\nabla p_{h}, \nabla q\right)_{K^{\prime}} \quad-\delta \sum_{K \in K_{h}}\left(u_{h}, \nabla q\right)_{K}
$$

As (3.11), we also have

$$
E_{i+1}^{P}=\left(I-\tau\left(\left(B^{T}-\lambda_{h} F\right)\left(A-\lambda_{h} E\right)^{-1} B+\delta D_{1}\right)\right) E_{i}^{P} .
$$

Method 3 (Multiscale enrichment method). Find $\left(u_{h}, p_{h} ; \lambda_{h}\right) \in\left(X_{h} \times M_{h}\right) \times \mathbb{R}$ with $\left\|u_{h}\right\|_{0}=1$, such that for all $(v, q) \in X_{h} \times M_{h}$,

$$
B\left(\left(u_{h}, p_{h}\right) ;(v, q)\right)-\delta_{1} \sum_{K \in K_{h}}\left(\nabla p_{h}-\lambda_{h} u_{h}, \nabla q\right)_{K}+\delta_{2} \sum_{e=\partial K_{j} \cap \partial K_{k}}\left(\left[v \partial_{n} u_{h}\right],\left[v \partial_{n} v\right]\right)_{e}=\lambda_{h}\left(u_{h}, v\right)
$$

where $\delta_{1}=h^{2} /\left(\alpha_{1} v\right), \delta_{2}=h /\left(\alpha_{2} v\right)$ are the stabilization parameters, $\alpha_{1}, \alpha_{2}>0, n$ is the normal outward vector, $\partial_{n}$ is normal derivative operator, and $[v]$ denotes the jump of $v$ across $e$. This stabilized method includes the usual Galerkin least squares stabilized terms on each finite element and positive jump terms at interelement boundaries. Moreover, a direct algebraic manipulation leads to the matrix form

$$
\left[\begin{array}{cc}
A & B \\
B^{T}+\delta_{2} D_{2} & -\delta_{1} D_{1}
\end{array}\right]\left[\begin{array}{l}
U \\
P
\end{array}\right]=\lambda_{h}\left[\begin{array}{ll}
E & 0 \\
F & 0
\end{array}\right]\left[\begin{array}{l}
U \\
P
\end{array}\right]
$$

where the matrix $D_{2}$ is deduced in the usual manner, using the bases for $X_{h}$, from the term $\sum_{e=\partial K_{j} \cap \partial K_{k}}\left(\left[v \partial_{n} u_{h}\right],\left[v \partial_{n} v\right]\right)_{e}$. As (3.11), we have

$$
E_{i+1}^{P}=\left(I-\tau\left(\left(B^{T}+\delta_{2} D_{2}-\lambda_{h} F\right)\left(A-\lambda_{h} E\right)^{-1} B+\delta D_{1}\right)\right) E_{i}^{P}
$$

Method 4 (Local Gauss integration method). Find $\left(u_{h}, p_{h} ; \lambda_{h}\right) \in\left(X_{h} \times M_{h}\right) \times \mathbb{R}$ with $\left\|u_{h}\right\|_{0}=1$, such that for all $(v, q) \in X_{h} \times M_{h}$,

$$
B\left(\left(u_{h}, p_{h}\right) ;(v, q)\right)-G\left(p_{h}, q\right)=\lambda_{h}\left(u_{h}, v\right)
$$

where $G\left(p_{h}, q\right)$ is defined by

$$
G\left(p_{h}, q\right)=\sum_{K \in K_{h}}\left\{\int_{K, 2} p_{h} q \mathrm{~d} x-\int_{K, 1} p_{h} q \mathrm{~d} x\right\}, \quad \forall p_{h}, q \in M_{h},
$$

where $\int_{K, i} g(x) \mathrm{d} x$ indicates a local Gauss integral over $K$ that is exact for polynomials of degree $i, i=1$, 2 . In particular, the trial function $p_{h} \in M_{h}$ must be projected to piecewise 
constant space when $i=1$. This stabilization technique is free of stabilization parameters and does not require any calculation of high-order derivatives, a specification of any meshdependent parameter or edge-based data structures. Then the corresponding matrix form of this stabilized method is

$$
\left[\begin{array}{cc}
A & B \\
B^{T} & -G
\end{array}\right]\left[\begin{array}{l}
U \\
P
\end{array}\right]=\lambda_{h}\left[\begin{array}{ll}
E & 0 \\
0 & 0
\end{array}\right]\left[\begin{array}{l}
U \\
P
\end{array}\right]
$$

where the matrix $G$ is deduced in the usual manner, using the bases for $M_{h}$, from the term $G\left(p_{h}, q\right)$. As (3.11), we get

$$
E_{i+1}^{P}=\left(I-\tau\left(B^{T}\left(A-\lambda_{h} E\right)^{-1} B+G\right)\right) E_{i}^{P} .
$$

Remark 3.3. It is well known that if $\tau$ is well chosen, then $U_{i}$ and $P_{i}$ converge, respectively, to $U$ and $P$ with a rate of convergence based on (3.11), (3.15), (3.18), and (3.22). From these equations, we can find that Method 1 converges faster than Method 4. Methods 2 and 3, whose coefficient matrices are not symmetric, may cost more time to converge.

Remark 3.4. By using the regularity assumptions and well-established techniques for eigenvalue approximation $[1,8,10,12]$, the theoretical convergence rates should be of order $O\left(h^{2}\right)$ and $O(h)$ for the velocity in the $L^{2}$ - and $H^{1}$-norms, respectively, of order $O(h)$ for the pressure in the $L^{2}$-norm, and of order $O\left(h^{2}\right)$ for the eigenvalue by using all these stabilized methods.

\section{Numerical Experiments}

In this section we numerically compare the performance of the various stabilized mixed finite element methods discussed in the previous section. In all experiments, the algorithms are implemented using public domain finite element software [27] with some of our additional codes.

Let the computation be carried out in the region $\Omega=\{(x, y) \mid 0<x, y<1\}$. We consider the Stokes eigenvalue problem in the case of the viscidity $v=1$, and it will be numerically solved by the stabilized mixed methods on uniform mesh (see Figure 1). Here, we just consider the first eigenvalue of the Stokes eigenvalue problem for the sake of simplicity. The exact solution of this problem is unknown. Thus, we take the numerical solution by the standard Galerkin method ( $P_{2}-P_{1}$ element) computed on a very fine mesh (6742 grid points) as the "exact" solution for the purpose of comparison. Here, we take $\lambda=52.3447$ as the first exact eigenvalue.

As we know, the stabilized term of the regular and multiscale enrichment methods must be controlled by carefully designed stabilization parameters (i.e., $\delta, \delta_{1}, \delta_{2}$ ), whose optimal values are often unknown. Hence, in Figures 2 and 3 we show the effect on the error of varying $\delta, \delta_{1}$, and $\delta_{2}$ on a fixed mesh $h=1 / 64$ for the regular and multiscale enrichment approximations, respectively. An interesting thing can be observed that these two methods have an analogous convergence pattern with respect to the parameter $\delta$ and $\delta_{1}$. Because they involve a similar stabilization term with respect to these two parameters, we note that the errors can become large with some values of the stabilization parameters. 


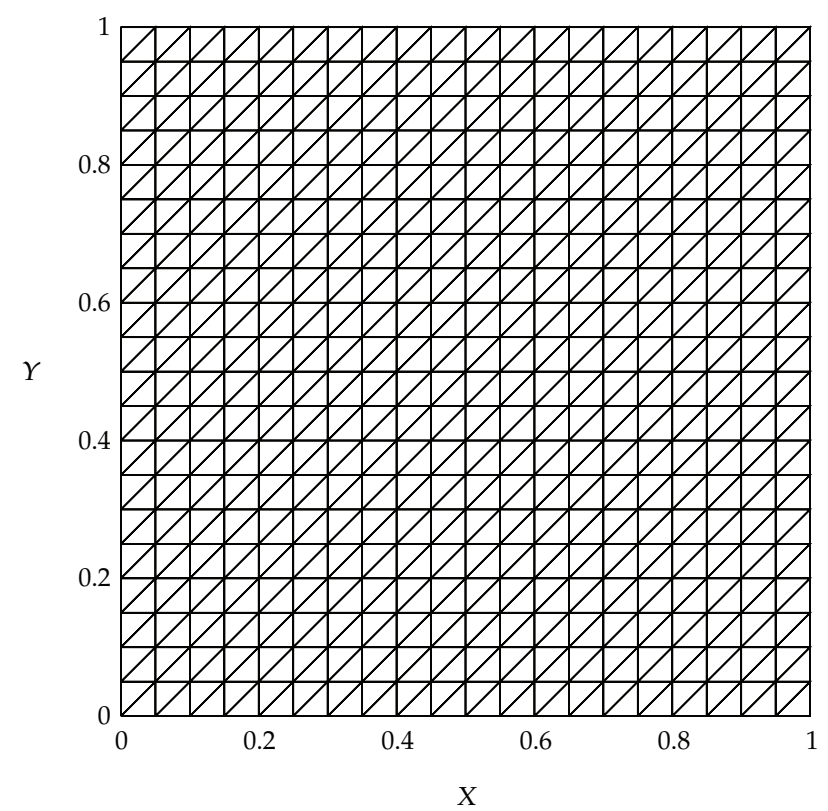

Figure 1: Uniform finite element partitioning of the unit square.

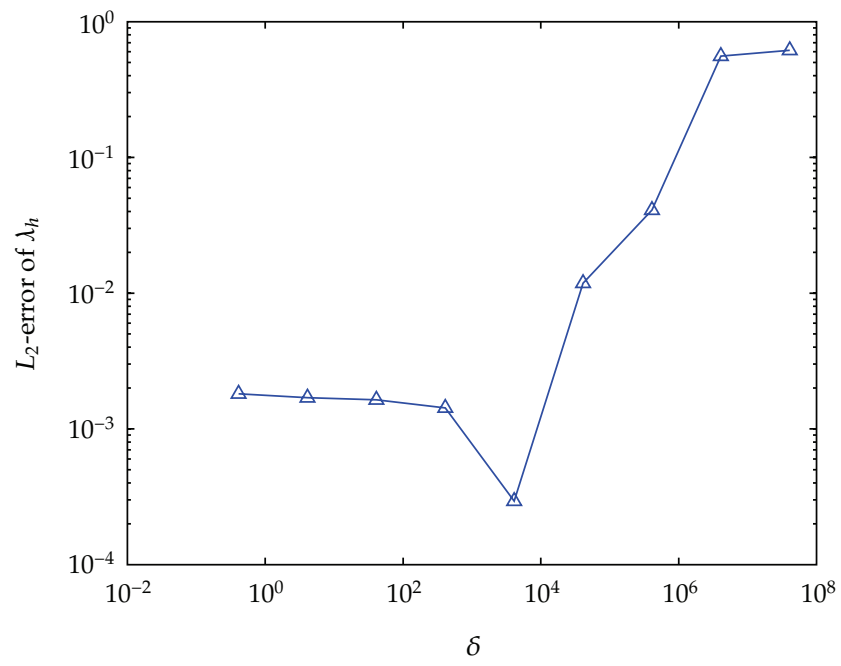

Figure 2: Effect of varying $\delta$ for the regular method.

Results gotten from the penalty, regular, multiscale enrichment, local Gauss integration, and nonconforming local Gauss integration methods are presented in Tables 1-5, respectively. Here, we choose $\varepsilon=1.0 e-5, \alpha=8, \alpha_{1}=8$, and $\alpha_{2}=12$. Because they can deal with the considered problem well. From Tables 1, 2, 3, 4, and 5, we can see that these methods work well and keep the convergence rates just like the theoretical analysis except the multiscale enrichment method. Meanwhile, it can be seen that the penalty method requires the least CPU-time, which validates the analysis in Remark 3.3. As expected, we have an 


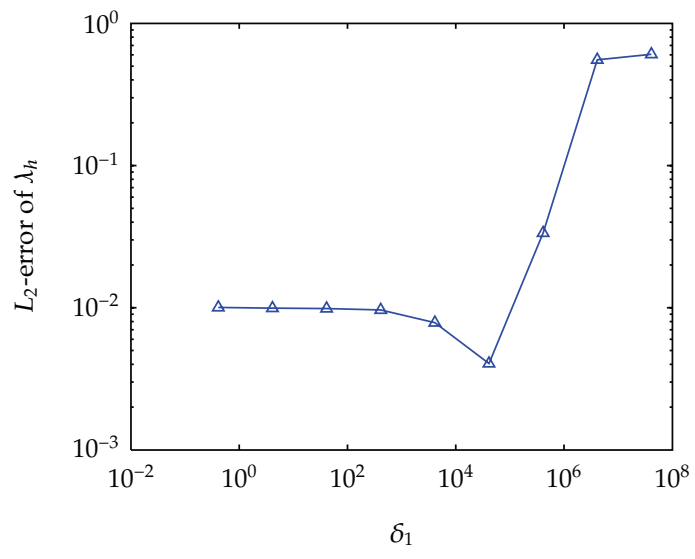

(a)

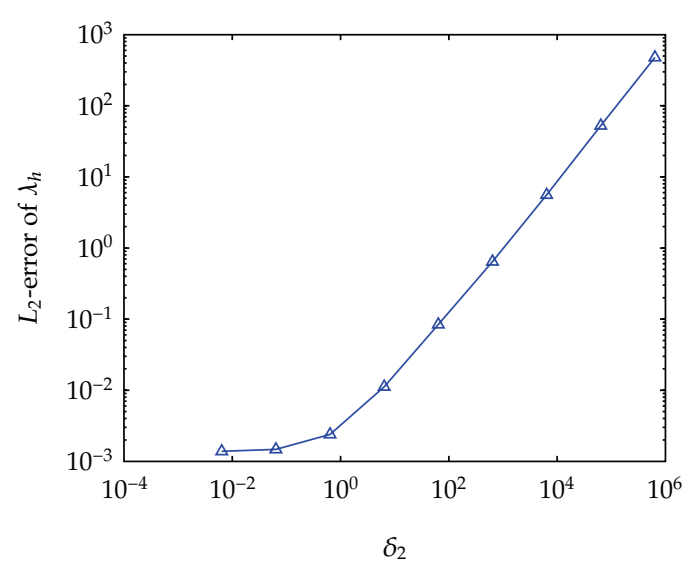

(b)

Figure 3: Effect of varying $\delta_{1}(\mathrm{a})$ and $\delta_{2}(\mathrm{~b})$ for the multiscale enrichment method.

Table 1: Results get from the penalty method with $\varepsilon=1.0 e-5$.

\begin{tabular}{lcccr}
\hline $1 / h$ & CPU-time & $\lambda_{h}$ & $\left|\lambda_{h}-\lambda\right| / \lambda$ & Rate \\
\hline 8 & 0.016 & 60.2628 & 0.151269 & - \\
16 & 0.094 & 54.1688 & 0.0348472 & 2.1180 \\
24 & 0.234 & 53.1426 & 0.015243 & 2.0393 \\
32 & 0.437 & 52.7909 & 0.00852367 & 2.0205 \\
40 & 0.751 & 52.6295 & 0.00544061 & 2.0120 \\
48 & 52.5433 & 0.00377371 & 2.0065 \\
56 & 1.156 & 52.4898 & 0.00277154 & 2.0023 \\
64 & 1.734 & 52.4558 & 0.00212237 & 1.9986 \\
\hline
\end{tabular}

Table 2: Results get from the regular method with $\alpha=8$.

\begin{tabular}{lcccc}
\hline $1 / h$ & CPU-time & $\lambda_{h}$ & $\left|\lambda_{h}-\lambda\right| / \lambda$ & Rate \\
\hline 8 & 0.031 & 56.7283 & 0.0837442 & - \\
16 & 0.109 & 53.4803 & 0.0216951 & 1.9486 \\
24 & 0.266 & 52.8531 & 0.00971199 & 1.9822 \\
32 & 0.515 & 52.6314 & 0.00547673 & 1.9913 \\
40 & 52.5284 & 0.00350903 & 1.9950 \\
48 & 0.861 & 52.4723 & 0.00243822 & 1.9969 \\
56 & 1.313 & 52.4385 & 0.00179192 & 1.9979 \\
64 & 1.953 & 52.4165 & 0.00137219 & 1.9986 \\
\hline
\end{tabular}

interesting observation that the error of the nonconforming local Gauss integration method is better than the conforming version, which is not surprising since the degrees of freedom of the nonconforming method are nearly three times than that of conforming one on uniform mesh (see Figure 1). Hence, it is natural that the nonconforming local Gauss integration method is more accurate and costs more CPU-time.

Besides, to show the stability and efficiency of these methods for the considered problem, we present the velocity streamlines and the pressure contours with $h=1 / 64$ 
Table 3: Results get from the multiscale enrichment method with $\alpha_{1}=8$ and $\alpha_{2}=12$.

\begin{tabular}{lcccc}
\hline $1 / h$ & CPU-time & $\lambda_{h}$ & $\left|\lambda_{h}-\lambda\right| / \lambda$ & Rate \\
\hline 8 & 0.063 & 67.8237 & 0.295713 & - \\
16 & 0.281 & 57.1062 & 0.0909634 & 1.7008 \\
24 & 0.656 & 54.7378 & 0.0457175 & 1.6968 \\
32 & 1.265 & 53.8304 & 0.0283839 & 1.6569 \\
40 & 2.156 & 53.3813 & 0.0198027 & 1.6133 \\
48 & 3.359 & 53.1229 & 0.0148665 & 1.5725 \\
56 & 5.125 & 52.9588 & 0.0117327 & 1.5357 \\
64 & 7.187 & 52.8472 & 0.00959935 & 1.5029 \\
\hline
\end{tabular}

Table 4: Results get from the local Gauss integration method.

\begin{tabular}{lcccc}
\hline $1 / h$ & CPU-time & $\lambda_{h}$ & $\left|\lambda_{h}-\lambda\right| / \lambda$ & Rate \\
\hline 8 & 0.031 & 57.3951 & 0.096482 & - \\
16 & 0.109 & 53.6201 & 0.024366 & 1.9854 \\
24 & 0.251 & 52.9119 & 0.0108368 & 1.9983 \\
32 & 0.469 & 52.6638 & 0.00609553 & 2.0001 \\
40 & 0.797 & 52.5489 & 0.00390065 & 2.0006 \\
48 & 1.234 & 52.4865 & 0.00270843 & 2.0007 \\
56 & 1.844 & 52.4488 & 0.00198963 & 2.0008 \\
64 & 2.547 & 52.4244 & 0.00152315 & 2.0008 \\
\hline
\end{tabular}

Table 5: Results get from the local Gauss integration method with the nonconforming element.

\begin{tabular}{lcccc}
\hline $1 / h$ & CPU-time & $\lambda_{h}$ & $\left|\lambda_{h}-\lambda\right| / \lambda$ & Rate \\
\hline 8 & 0.031 & 50.2121 & 0.0407434 & - \\
16 & 0.125 & 51.7355 & 0.0116391 & 1.8076 \\
24 & 0.344 & 52.0619 & 0.00540181 & 1.8932 \\
32 & 52.1825 & 0.00309932 & 1.9311 \\
40 & 0.672 & 52.2397 & 0.00200547 & 1.9508 \\
48 & 1.156 & 52.2713 & 0.00140228 & 1.9624 \\
56 & 1.859 & 52.2905 & 0.00103505 & 1.9698 \\
64 & 2.797 & 52.3031 & 0.000795121 & 1.9749 \\
\hline
\end{tabular}

in Figures 5, 6, 7, 8, and 9. Meanwhile, we present the results by the standard Galerkin method ( $P_{2}-P_{1}$ element) computed on a very fine mesh (6742 grid points) for the purpose of comparison in Figure 4. From Figures 5(a)-9(a), five resolved vortices are captured, which is consistent with that in Figure 4(a). For the pressure, the penalty method is divergent from Figure 5(b), although it costs the least time. From Figures 6(b)-9(b), the nonconforming local Gauss integration method shows the best numerical stability.

\section{Conclusions}

In this paper we have presented several stabilized mixed finite element methods in solving the Stokes eigenvalue problem based on the lowest equal-order finite element space pair. By being compared numerically, we get some conclusions as follows. 


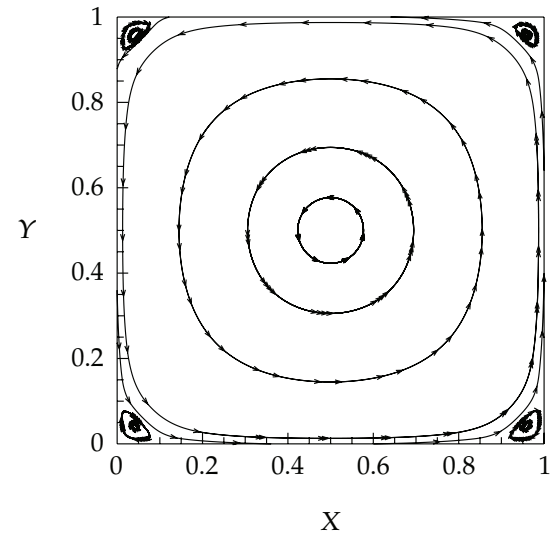

(a)

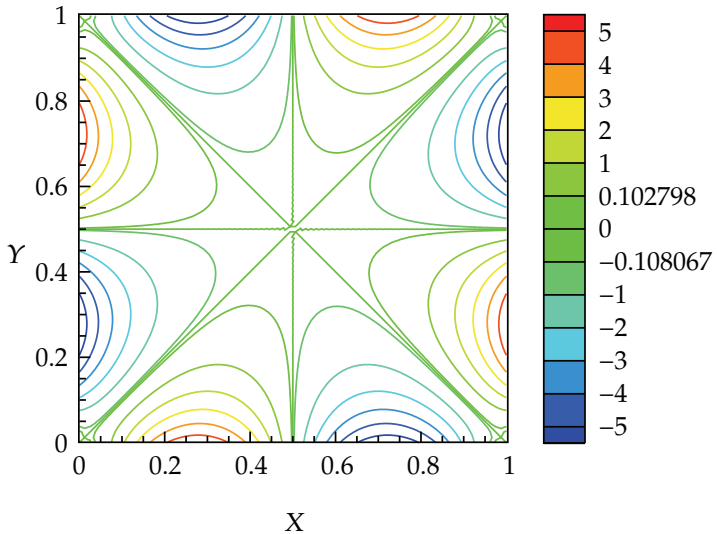

(b)

Figure 4: Velocity streamlines (a) and pressure level lines (b) for the standard Galerkin method $\left(P_{2}-P_{1}\right.$ element).

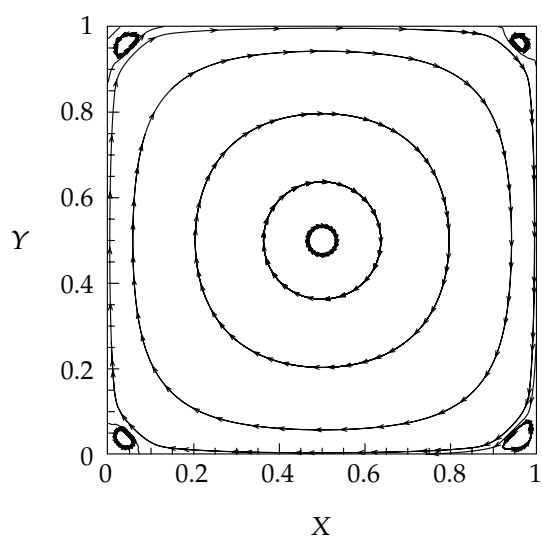

(a)

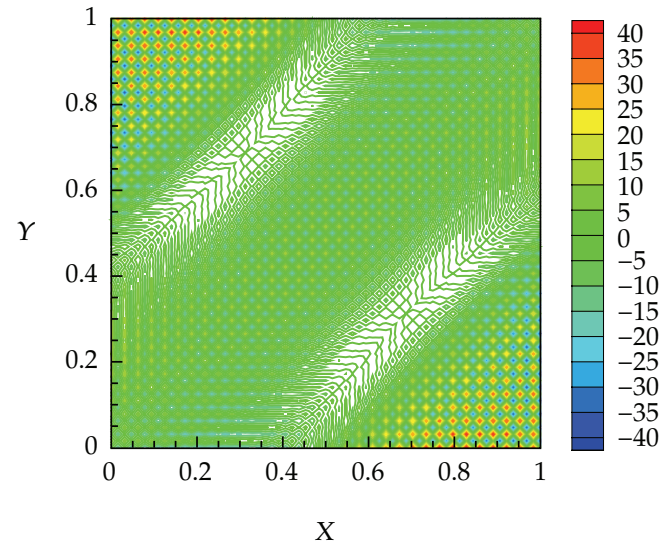

(b)

Figure 5: Velocity streamlines (a) and pressure level lines (b) for the penalty method with $\varepsilon=1.0 e-5$.

(i) The stability and efficiency of the penalty method depend on the penalty parameter. The smaller this parameter, the more stable the method. However, if this parameter is too small, the condition number of the system matrix arising from this method will become too large to solve.

(ii) The performance of the regular and multiscale enrichment method heavily depends on the choice of the stabilization parameters, which is a difficult task in reality. Meanwhile, a poor choice of these stabilization parameters can also lead to serious deterioration in the convergence rates.

(iii) The local integration method is free of stabilization parameters and shows numerically the best performance among the methods considered for the given problem. 


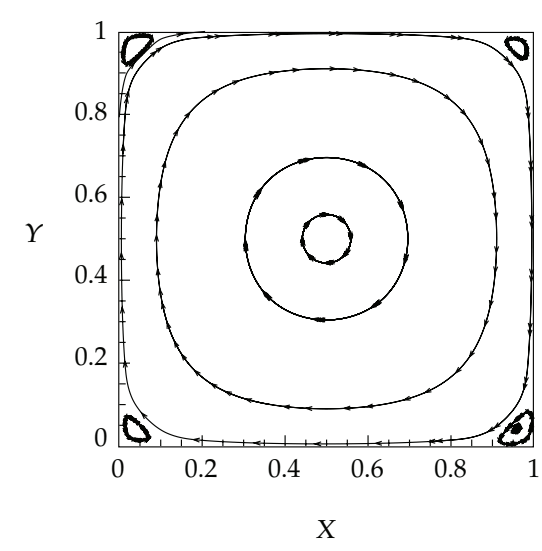

(a)

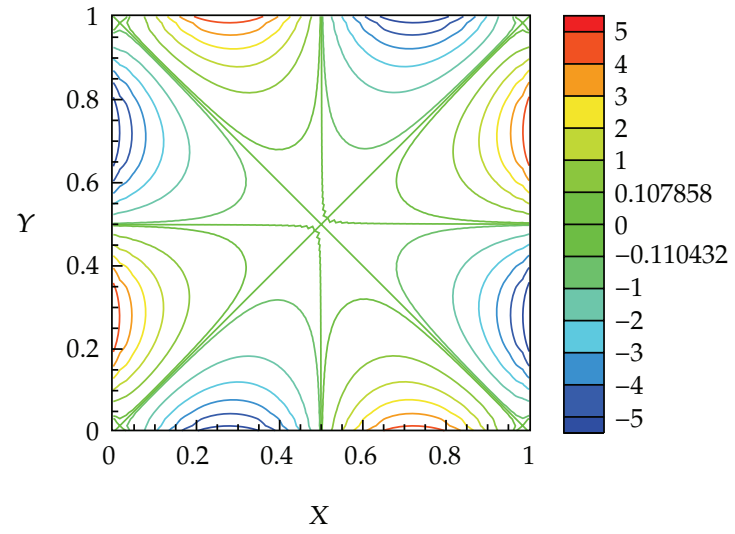

(b)

Figure 6: Velocity streamlines (a) and pressure level lines (b) for the regular method with $\alpha=8$.

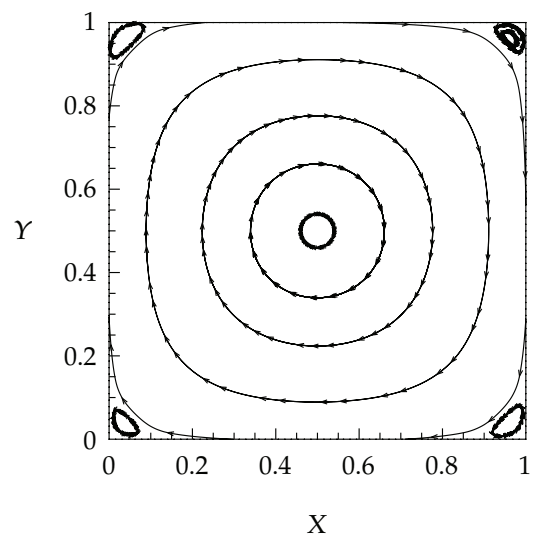

(a)

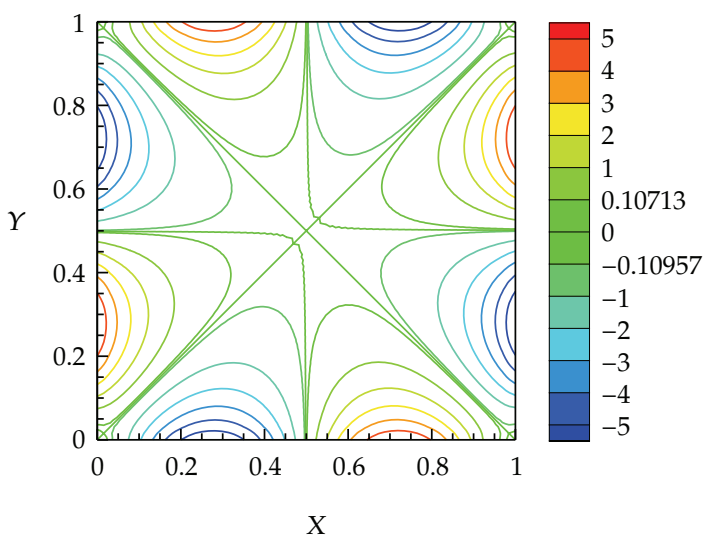

(b)

Figure 7: Velocity streamlines (a) and pressure level lines (b) for the multiscale enrichment method with $\alpha_{1}=8$ and $\alpha_{2}=12$.

(iv) From Tables 4 and 5, we have an interesting observation that the value of $\lambda_{h}$ by conforming method becomes small to converge to the exact solution and the one by nonconforming method becomes large to converge to the exact solution. We hold that it can obtain more accurate numerical solution by Lagrange interpolation between conforming and nonconforming results based on the same degrees of freedom of these two methods. It may get superconvergence result on this triangular mesh.

\section{Acknowledgments}

The authors would like to thank the editor and reviewers for their valuable comments and suggestions which helped us improve the results of this paper. This work is in parts supported by the NSF of China (no. 10901131, no. 10971166), the National High Technology 


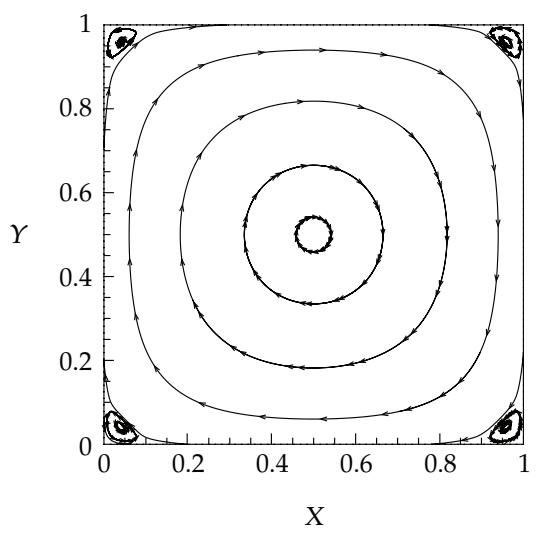

(a)

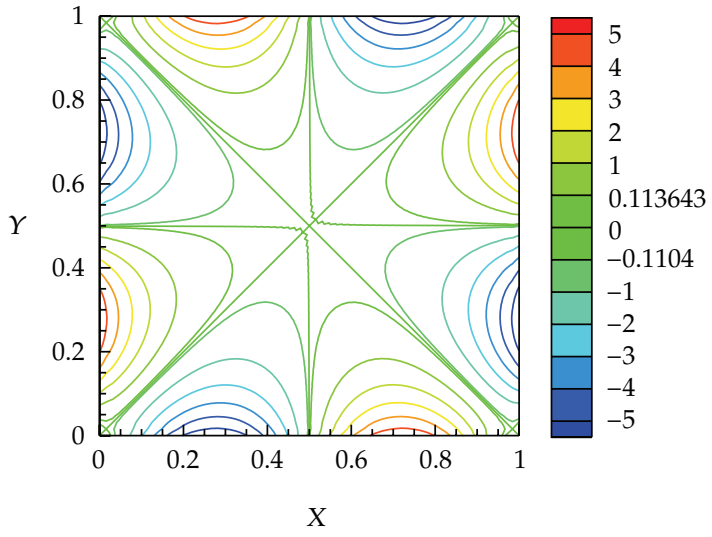

(b)

Figure 8: Velocity streamlines (a) and pressure level lines (b) for the local Gauss integration method.

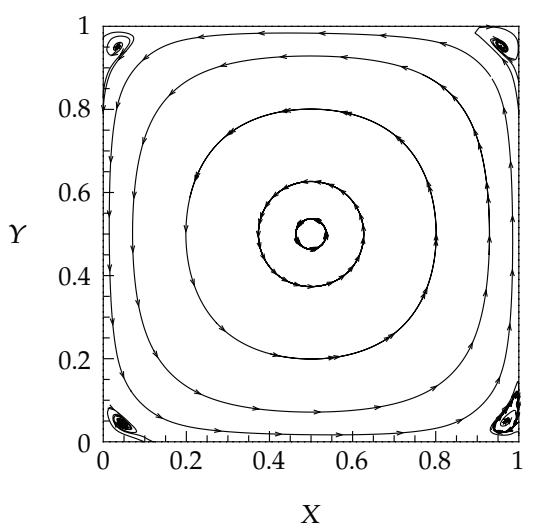

(a)

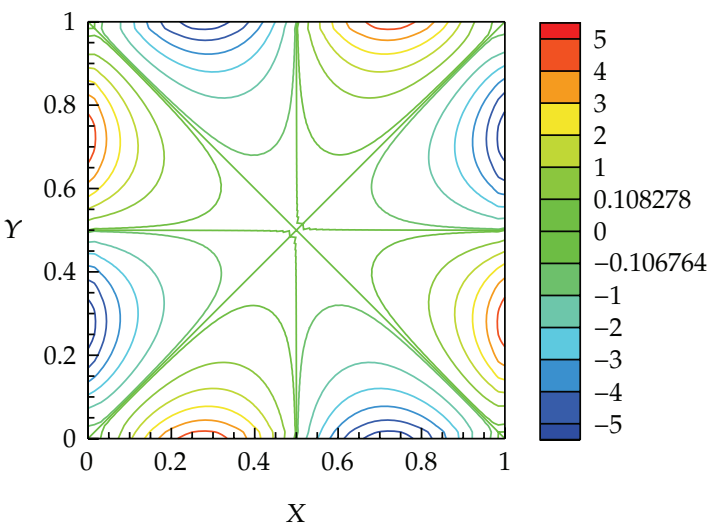

(b)

Figure 9: Velocity streamlines (a) and pressure level lines (b) for the local Gauss integration method with the nonconforming element.

Research and Development Program of China (863 Program, no. 2009AA01A135), the China Postdoctoral Science Foundation (no. 200801448, no. 20070421155), and the Natural Science Foundation of Xinjiang Province (no. 2010211B04).

\section{References}

[1] I. Babuška and J. E. Osborn, "Eigenvalue problems," in Handbook of Numerical Analysis, P. G. Ciarlet and J. L. Lions, Eds., vol. II, pp. 641-787, Finite Element Method, North-Holland, Amsterdam, 1991.

[2] I. Babuška and J. E. Osborn, "Finite element-Galerkin approximation of the eigenvalues and eigenvectors of selfadjoint problems," Mathematics of Computation, vol. 52, no. 186, pp. 275-297, 1989.

[3] Q. Lin and H. Xie, "Asymptotic error expansion and Richardson extrapolation of eigenvalue approximations for second order elliptic problems by the mixed finite element method," Applied Numerical Mathematics, vol. 59, no. 8, pp. 1884-1893, 2009. 
[4] Q. Lin, "Fourth order eigenvalue approximation by extrapolation on domains with reentrant corners," Numerische Mathematik, vol. 58, no. 6, pp. 631-640, 1991.

[5] S. Jia, H. Xie, X. Yin, and S. Gao, "Approximation and eigenvalue extrapolation of Stokes eigenvalue problem by nonconforming finite element methods," Applications of Mathematics, vol. 54, no. 1, pp. 1$15,2009$.

[6] H. Chen, S. Jia, and H. Xie, "Postprocessing and higher order convergence for the mixed finite element approximations of the eigenvalue problem," Applied Numerical Mathematics, vol. 61, no. 4, pp. 615-629, 2011.

[7] H. Chen, S. Jia, and H. Xie, "Postprocessing and higher order convergence for the mixed finite element approximations of the Stokes eigenvalue problems," Applications of Mathematics, vol. 54, no. 3, pp. 237250, 2009.

[8] C. Lovadina, M. Lyly, and R. Stenberg, "A posteriori estimates for the Stokes eigenvalue problem," Numerical Methods for Partial Differential Equations, vol. 25, no. 1, pp. 244-257, 2009.

[9] K. A. Cliffe, E. Hall, and P. Houston, "Adaptive discontinuous Galerkin methods for eigenvalue problems arising in incompressible fluid flows," SIAM Journal on Scientific Computing, vol. 31, no. 6, pp. 4607-4632, 2010.

[10] X. Yin, H. Xie, S. Jia, and S. Gao, "Asymptotic expansions and extrapolations of eigenvalues for the Stokes problem by mixed finite element methods," Journal of Computational and Applied Mathematics, vol. 215, no. 1, pp. 127-141, 2008.

[11] W. Chen and Q. Lin, "Approximation of an eigenvalue problem associated with the Stokes problem by the stream function-vorticity-pressure method," Applications of Mathematics, vol. 51, no. 1, pp. 73$88,2006$.

[12] B. Mercier, J. Osborn, J. Rappaz, and P. A. Raviart, “Eigenvalue approximation by mixed and hybrid methods," Mathematics of Computation, vol. 36, no. 154, pp. 427-453, 1981.

[13] J. C. Xu and A. H. Zhou, "A two-grid discretization scheme for eigenvalue problems," Mathematics of Computation, vol. 70, no. 233, pp. 17-25, 2009.

[14] Z. X. Chen, Finite Element Methods and Their Applications, Springer, Heidelberg, Germany, 2005.

[15] V. Girault and P. A. Raviart, Finite Element Method for Navier-Stokes Equations: Theory and Algorithms, Springer, Berlin, Germany, 1987.

[16] B. Brefort, J. M. Ghidaglia, and R. Temam, "Attractors for the penalized Navier-Stokes equations," SIAM Journal on Mathematical Analysis, vol. 19, no. 1, pp. 1-21, 2001.

[17] Y. N. He, "Optimal error estimate of the penalty finite element method for the time-dependent NavierStokes equations," Mathematics of Computation, vol. 74, no. 251, pp. 1201-1216, 2005.

[18] J. Li, L. Q. Mei, and Y. N. He, "A pressure-Poisson stabilized finite element method for the non-stationary Stokes equations to circumvent the inf-sup condition," Applied Mathematics and Computation, vol. 182, no. 1, pp. 24-35, 2006.

[19] J. Douglas Jr. and J. Wang, "An absolutely stabilized finite element method for the Stokes problem," Mathematics of Computation, vol. 52, no. 186, pp. 495-508, 1989.

[20] R. Araya, G. R. Barrenechea, and F. Valentin, "Stabilized finite element methods based on multiscale enrichment for the stokes problem," SIAM Journal on Numerical Analysis, vol. 44, no. 1, pp. 322-348, 2006.

[21] J. Li, Y. N. He, and Z. X. Chen, "A new stabilized finite element method for the transient Navier-Stokes equations," Computer Methods in Applied Mechanics and Engineering, vol. 197, no. 1-4, pp. 22-35, 2007.

[22] J. Li and Y. N. He, "A stabilized finite element method based on two local Gauss integrations for the Stokes equations," Journal of Computational and Applied Mathematics, vol. 214, no. 1, pp. 58-65, 2008.

[23] X. L. Feng, I. Kim, H. Nam, and D. Sheen, "Locally stabilized $P_{1}$-nonconforming quadrilateral and hexahedral finite element methods for the Stokes equations," Journal of Computational and Applied Mathematics. In press.

[24] J. Li, Y. N. He, and Z. X. Chen, "Performance of several stabilized finite element methods for the Stokes equations based on the lowest equal-order pairs," Computing, vol. 86, no. 1, pp. 37-51, 2009.

[25] F. Brezzi and M. Fortin, Mixed and Hybrid Finite Element Methods, vol. 15, Springer, New York, NY, USA, 1991, Springer Series in Computational Mathematics.

[26] J. Li and Z. X. Chen, "A new local stabilized nonconforming finite element method for the Stokes equations," Computing, vol. 82, no. 2-3, pp. 157-170, 2008.

[27] FreeFem++, version 2.23, http:/ /www.freefem.org/. 


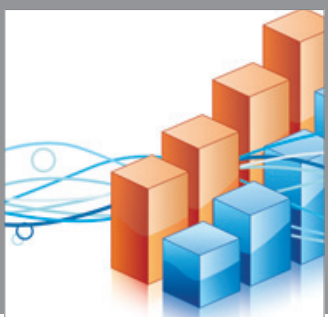

Advances in

Operations Research

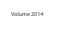

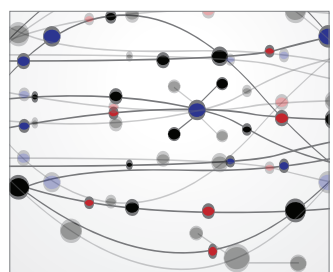

\section{The Scientific} World Journal
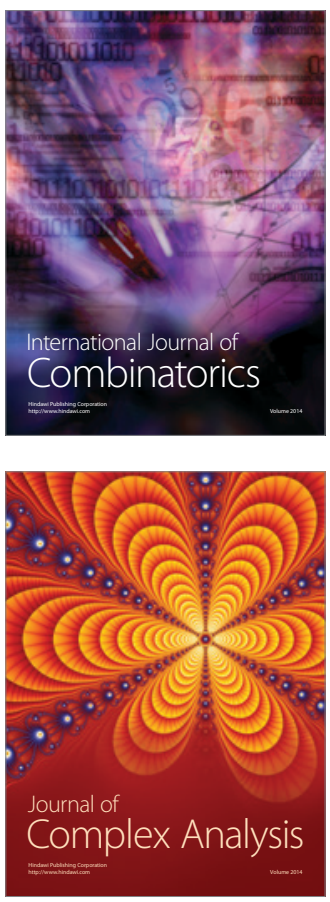

International Journal of

Mathematics and

Mathematical

Sciences
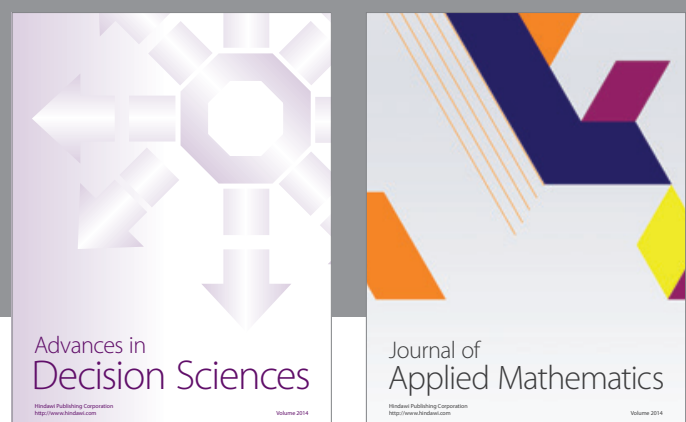

Journal of

Applied Mathematics
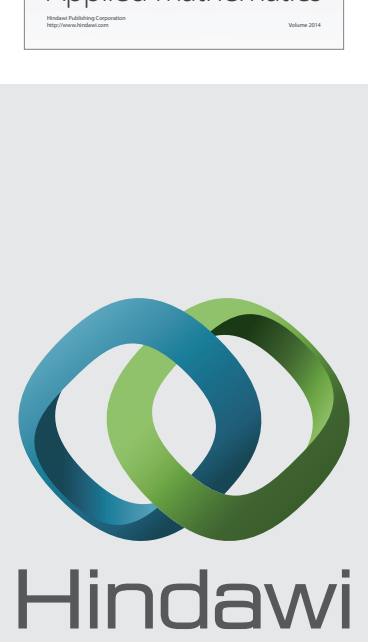

Submit your manuscripts at http://www.hindawi.com
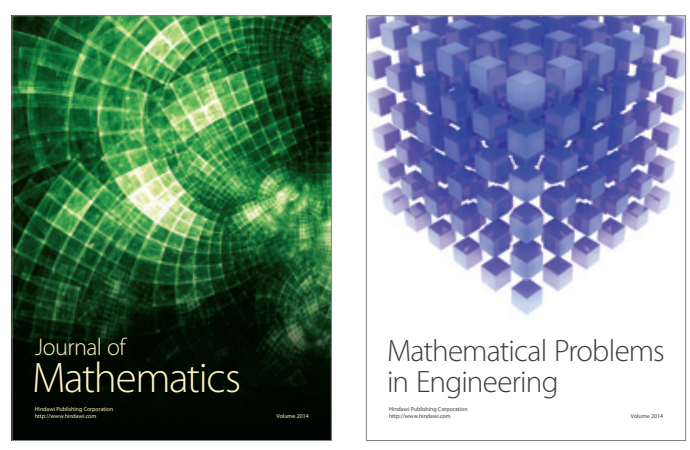

Mathematical Problems in Engineering
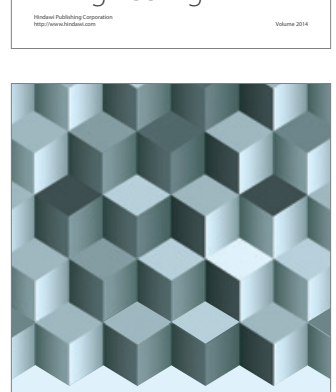

Journal of

Function Spaces
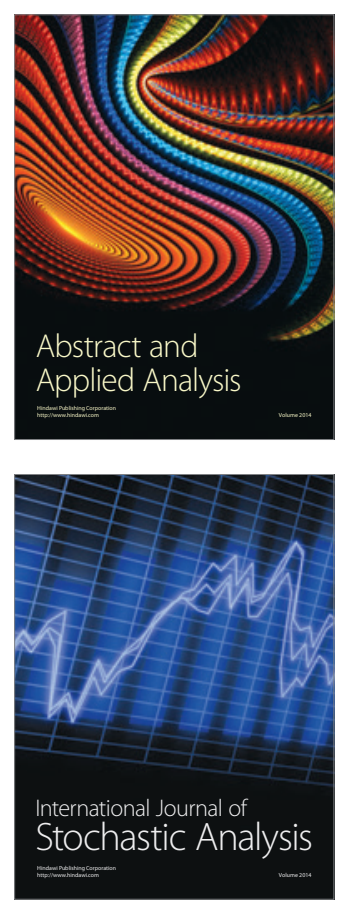

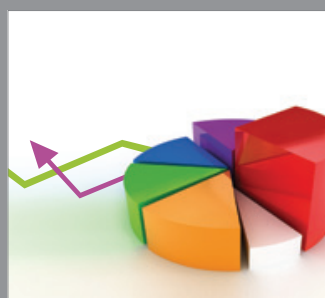

ournal of

Probability and Statistics

Promensencen
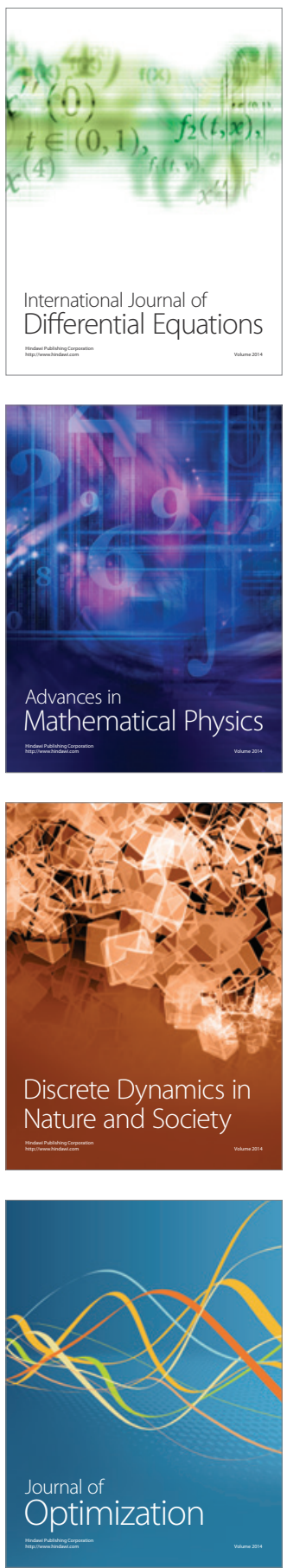